\title{
Inhibition of 6-hydroxydopamine-induced endoplasmic reticulum stress by sulforaphane through the activation of Nrf2 nuclear translocation
}

\author{
CHANG DENG ${ }^{1}$, RAN TAO ${ }^{2}$, SHUN-ZHI YU ${ }^{3}$ and HUA JIN ${ }^{4}$ \\ ${ }^{1}$ Deparment of Emergency, the People's Hospital of Guang Du District, Kunming 650200; \\ ${ }^{2}$ The Second Deparment of Internal Medicine, the People's Hospital of Panlong District, Kunming 650021; \\ ${ }^{3}$ Mental Health Hospital of Prevention and Treatment in Kunming City, Kunming 650041; ${ }^{4}$ Department of Anesthesiology, \\ Kunhua Hospital, the First People's Hospital of Yunnan Province, Kunming 650032, P.R. China
}

Received January 9, 2012; Accepted March 30, 2012

DOI: $10.3892 / \mathrm{mmr} .2012 .894$

\begin{abstract}
Endoplasmic reticulum (ER) stress plays a key role in the development of neurodegenerative diseases, including Parkinson's disease (PD). Sulforaphane (SF) is a natural drug derived from isothiocyanate found in cruciferous vegetables. Although there are reports indicating that SF is a potential candidate for PD treatment, there have been no reports on the effects of SF on ER stress in PD. In this study, we investigated the cytoprotective effects of SF on 6-hydroxydopamine (6-OHDA)induced ER stress in rat PC12 cells. Pre-treatment with SF elicited cytoprotection against 6-OHDA-induced cytotoxicity. Consistent with its cytoprotective action, SF significantly inhibited subsequent ER stress, including the expression of Bip and the $\mathrm{C} / \mathrm{EBP}$ homologous protein. We also found that transfection with NF-E2-related factor-2 (Nrf2) siRNA reversed the inhibitory effects of SF on 6-OHDA-induced ER stress responses. In conclusion, our results show that $\mathrm{SF}$ can prevent ER stress response induced by 6-OHDA through the activation of $\mathrm{Nrf} 2$. SF may be a therapeutic candidate for the treatment of ER stress-associated neural diseases, including PD.
\end{abstract}

\section{Introduction}

Parkinson's disease (PD) is the second most common neurodegenerative disorder and is characterized by the loss of nigrostriatal dopaminergic neurons and the presence of Lewy bodies in the neurons (1). Although PD has been widely studied, the pathogenesis of PD remains unknown. There are no effective therapies available to prevent PD progression and neurodegeneration. However, significant clues into the

Correspondence to: Dr Hua Jin, Department of Anesthesiology, Kunhua Hospital, the First People's Hospital of Yunnan Province, Kunming 650032, P.R. China

E-mail: jinghuakm@163.com

Key words: 6-hydroxydopamine, endoplasmic reticulum, sulforaphane, Parkinson's disease pathogenesis of PD have been yielded by the use of 6-hydroxydopamine (6-OHDA), 1-methyl-4-phenylpyridinium $\left(\mathrm{MPP}^{+}\right)$, 1-methyl-4-phenyl-1,2,3,6-tetrahydropyridine (MPTP) and rotenone (2-4). 6-OHDA is a selective catecholaminergic neurotoxin that replicates most of the neuropathological hallmarks of PD $(5,6)$. 6-OHDA is commonly used to produce an experimental model of PD in vitro or in vivo.

The endoplasmic reticulum (ER) is an important organelle involved in calcium signaling and the synthesis, folding and processing of proteins (7). Impaired function of the ER leads to ER stress, which is caused by oxidative stress, changes in $\mathrm{Ca}^{2+}$ homeostasis and accumulation of unfolded or misfolded proteins (8). ER stress activates signaling pathways, including the unfolded protein response (UPR) pathway (9). When ER stress occurs, the phosphorylation of eukaryotic initiation factor $2 \alpha(\mathrm{eIF}-2 \alpha)$ is firstly enhanced to reduce the load of newly synthesized proteins $(10,11)$. Then, ER chaperones, such as Bip, are induced to enhance the folding activity in the ER (12). However, if the function of the ER is severely impaired, the apoptotic pathway is activated to remove the damaged cells. One of the components of ER stress-mediated apoptosis is the $\mathrm{C} / \mathrm{EBP}$ homologous protein (CHOP), also known as growth arrest and DNA damage inducible gene 153 (GADD153). CHOP is expressed at low levels under physiological conditions, but is strongly induced in response to ER stress $(13,14)$. ER stress-induced apoptosis is suppressed in both CHOP-null cells and CHOP-knockout mice (15). ER stress-induced cellular dysfunction is critical in the development and progression of human diseases, including stroke, diabetes and neurodegeneration $(8,16)$.

Sulforaphane (SF) is a natural drug derived from isothiocyanate found in cruciferous vegetables, such as broccoli, cabbage and cauliflower. SF is known to have cytoprotective effects by activating the transcription factor, NF-E2-related factor-2 (Nrf2), which binds to the anti-oxidant response element in the promoter region of a number of genes encoding anti-oxidative and phase 2 enzymes, including heme oxygenase-1, glutathione reductase and $\mathrm{NAD}(\mathrm{P}) \mathrm{H}$ :quinoine oxidoreductase 1 (17-19). SF first gained attention due to its potential as an anticancer agent (20). Recent studies have indicated that SF can serve as 
a potential candidate for the development of treatments for and/or the prevention of PD (21). SF has been shown to protect dopaminergic neurons against 6-OHDA-induced cytotoxicity $(18,22,23)$. ER stress is a contributory factor to neuronal death and is involved in the pathogenesis of PD. A number of studies have shown how 6-OHDA induces dopaminergic neurons through mechanisms modulated by ER stress and UPR signaling components (4). However, there have been no reports on the involvement of SF in the modulation of ER stress. SF may act as a protective mechanism to attenuate ER stress, thus protecting cells from ER stress induced by 6-OHDA. In this study, to test this hypothesis, we measured the effects of SF on cell survival and ER stress responses induced by 6-OHDA in PC12 cells.

\section{Materials and methods}

Reagents. 6-OHDA, SF and 3-(4,5-dimethylthiazol-2-yl)2,5-diphenyl tetrazolium bromide (MTT) were obtained from Sigma Chemical Company (St. Louis, MO, USA). Antibodies against Bip, CHOP, $\beta$-actin, Lamin B and Nrf2 were purchased from Santa Cruz Biotechnology (Santa Cruz, CA, USA). Lipofectamine, RPMI-1640 medium, horse serum and fetal calf serum were obtained from Gibco-BRL/Life Technologies (Grand Island, NY, USA). All other chemicals were of the highest quality commercially available.

Cell culture. PC12 cells were cultured in RPMI-1640 medium supplemented with $10 \%$ heat-inactivated horse serum, $5 \%$ heat-inactivated fetal calf serum, $100 \mathrm{IU} / \mathrm{ml}$ penicillin and $100 \mu \mathrm{g} / \mathrm{ml}$ streptomycin in a humidified atmosphere of $5 \%$ $\mathrm{CO}_{2}$ in air at $37^{\circ} \mathrm{C}$.

MTT assay. For the determination of the cell viability, the conventional MTT assay was used. PC12 cells were pre-treated with SF for $6 \mathrm{~h}$, and then incubated with 6-OHDA. After exposure to 6-OHDA for $24 \mathrm{~h}$, the treated cells were incubated with $0.5 \mathrm{mg} / \mathrm{ml} \mathrm{MTT} \mathrm{for} 4 \mathrm{~h}$. Isopropyl alcohol containing $0.04 \mathrm{~N}$ hydrochloride (HCL) was added to the culture medium and mixed to dissolve dark blue crystals. The density was measured at $570 \mathrm{~nm}$ using a plate reader.

Nuclear and cytosolic lysate preparation. Nuclear and cytosolic extracts were isolated as follows. Cells were incubated in ice-cold buffer A. After 15 min of incubation on ice, NP-40 was added to a final concentration of $0.6 \%$, and then cells were vortexed and centrifuged for $1 \mathrm{~min}$ at $16,000 \mathrm{x}$ g. The nuclear pellet was extracted with buffer B [20 mM HEPES ( $\mathrm{pH} 8.0$ ), $1 \mathrm{mM}$ EDTA, $1.5 \mathrm{mM} \mathrm{MgCl} 2,10 \mathrm{mM} \mathrm{KCl}, 1 \mathrm{mM}$ DTT, $1 \mathrm{mM}$ sodium orthovanadate, $1 \mathrm{mM} \mathrm{NaF}, 1 \mathrm{mM}$ PMSF, $0.5 \mathrm{mg} / \mathrm{ml}$ benzamidine, $0.1 \mathrm{mg} / \mathrm{ml}$ leupeptin, $1.2 \mathrm{mg} / \mathrm{ml}$ aprotinin and $20 \%$ glycerol] for $30 \mathrm{~min}$ on ice. The debris was removed by centrifugation at $16,000 \mathrm{x}$ g for $20 \mathrm{~min}$ at $4^{\circ} \mathrm{C}$.

Western blot analysis. After treatment, cells were collected and washed twice with ice-cold phosphate-buffered saline (PBS). The harvested cells were then lysed with a solubilizing solution [20 mM Tris- $\mathrm{HCl}$ ( $\mathrm{pH}$ 7.0), $25 \mathrm{mM} \beta$-glycerophosphate, 2 mM EGTA, $1 \%$ Triton X-100, 1 mM vanadate, $1 \%$ aprotinin, $1 \mathrm{mM}$ phenylmethylsulfonyl fluoride and $2 \mathrm{mM}$ dithiothreitol] on ice for $40 \mathrm{~min}$. The lysate was centrifuged at 15,000 rpm for 15 min and supernatants were collected. Protein concentrations were determined using Bio-Rad protein assay reagent (Bio-Rad, Hercules, CA, USA). An equal quantity of proteins was separated by $10 \%$ SDS-polyacrylamide gel electrophoresis and transferred to a PVDF membrance (Millipore Corporation, Billerica, MA, USA). The membrances were incubated with Bip, CHOP, $\beta$-actin, Lamin B and Nrf2 antibodies, washed and then incubated with peroxidase-conjugated anti-rabbit or anti-mouse IgG (KPL, Gaithersburg, MD, USA). The immunoblot was revealed with an ECL Western Blot Detection kit (Amersham Pharmacia Biotech, Buckinghamshire, UK).

Transfection of siRNA. Rat siRNA against Nrf2 and scrambled control, non-silencing siRNA were purchased from Invitrogen. The target sequences for Nrf2-siRNA were 5'-UUA AGA CAC UGU AAC UCG GGA AUG G-3' (forward) and 5'-CCA UUC CCG AFU UAC AGU GUC UUA A-3' (reverse). The siRNA was transfected into PC12 cells at a concentration of $20 \mathrm{pmol} / 10^{5}$ cells by Lipofectamine $24 \mathrm{~h}$ prior to further experiments, according to the manufacturer's instructions.

Statistical analysis. All values are expressed as the means \pm SD. Data were analyzed by ANOVA followed by the Tukey-Kramer test as the post hoc test. Differences were considered statistically significant at a level of $\mathrm{P}<0.05$.

\section{Results}

Suppression of 6-OHDA-induced cell death by SF. To assess the effects of SF on 6-OHDA-induced cytotoxicity in PC12 cells, we first used MTT assay to examine the survival of cells treated with 6-OHDA and/or SF. As shown in Fig. 1, 6-OHDA $(80 \mu \mathrm{M})$ significantly induced cell death. Pre-treatment with SF $(0.1,1$ and $5 \mu \mathrm{M})$ inhibited 6-OHDA-induced cell death in a dose-dependent manner (Fig. 1).

SF inhibits 6-OHDA-induced ER stress. Previous studies have demonstrated that ER stress is a contributory factor in 6-OHDA-induced cytotoxicity. Thus, we investigated whether SF attenuates the ER stress response induced by 6-OHDA. Fig. 2 shows that 6-OHDA dramatically increased the expression of Bip and CHOP. Pre-treatment with SF significantly decreased these 6-OHDA-induced ER stress responses (Fig. 2). These results indicate that SF protects cells against 6-OHDA-induced cytotoxicity through the inhibition of cell death mediated by ER stress responses.

Inhibitory effects of SF on 6-OHDA-induced ER stress are reversed by silencing Nrf2 expression. A number of studies have shown that the protective effect of SF is dependent on the activation of the Nrf2/ARE pathway. To determine the signaling pathway involved in the inhibitory effects of SF on 6-OHDA-induced ER stress responses, we examined whether Nrf2 plays a role in these effects. As shown in Fig. 3A, SF induced the translocation of Nrf2 into the nucleus. In order to further elucidate the effect of Nrf2 activation on SF-mediated protective effects against 6-OHDA-induced ER stress, siRNA methodology was employed. Western blot analysis confirmed that $N r f 2$-specific siRNA significantly decreased the protein levels of Nrf2 (Fig. 3B). Notably, transfection of the Nrf2 


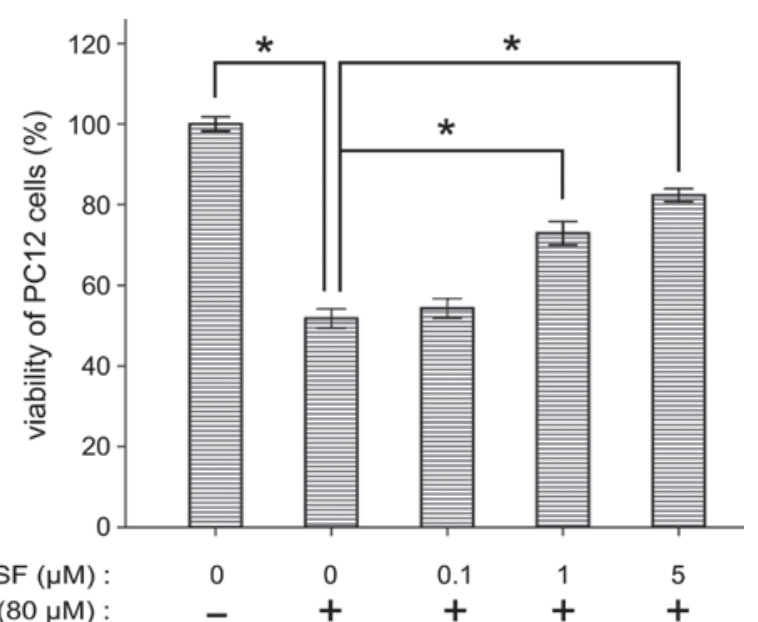

Figure 1. Effects of sulforaphane (SF) on the viability of cells treated with 6-hydroxydopamine (6-OHDA). PC12 cell were treated with SF $(0.1,1$ and $5 \mu \mathrm{M})$ for $6 \mathrm{~h}$ and then incubated with 6-OHDA $(80 \mu \mathrm{M})$ for a further $24 \mathrm{~h}$. Cell viability was measured with an MTT assay. ${ }^{*} \mathrm{P}<0.05$ indicates a statistically significant difference.

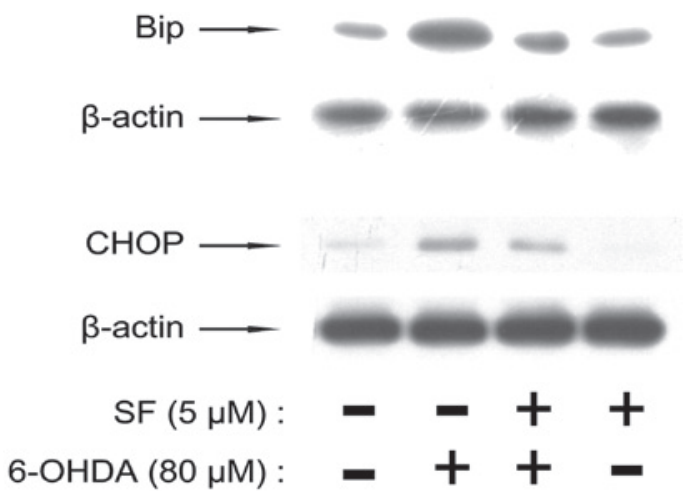

Figure 2. Expression of endoplasmic reticulum (ER) stress-related molecules in cells treated with 6-hydroxydopamine (6-OHDA) in the presence or absence of sulforaphane (SF). PC12 cells were pre-treated with $5 \mu \mathrm{M}$ SF for $6 \mathrm{~h}$ and then treated with $80 \mu \mathrm{M}$ 6-OHDA for $24 \mathrm{~h}$. Cells were harvested and lysed, and an aliquot of protein was subjected to SDS-PAGE for western blot analysis, as described in Materials and methods. Representative bands are shown from triplicate experiments. CHOP, C/EBP homologous protein.

siRNA reversed the inhibitory effects of SF on 6-OHDAinduced ER stress responses (Fig. 3C).

\section{Discussion}

Recent studies have shown that SF exhibits a broad spectrum of biological actions. Besides an anticarcinogen, SF may serve as a neuroprotective agent. A number of in vivo studies have demonstrated that SF protects against acute brain injury (19). SF protects immature hippocampal neurons against death caused by exposure to hemin or to oxygen and glucosedeprivation (24). SF has been shown to induce thioredoxin expression and attenuates retinal light damage in mice (25). It has also been reported that SF may serve as a potential candidate for the treatment and/or prevention of PD. SF causes the induction of $\mathrm{NAD}(\mathrm{P}) \mathrm{H}$ :quinoine oxidoreductase 1 and glutathione, removal of intracellular dopamine quinone and protects against 6-OHDA-induced toxicity $(18,22)$. To date, the beneficial bioactivities of SF have been attributed mainly

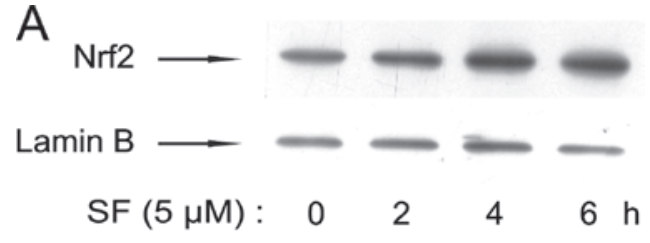

B
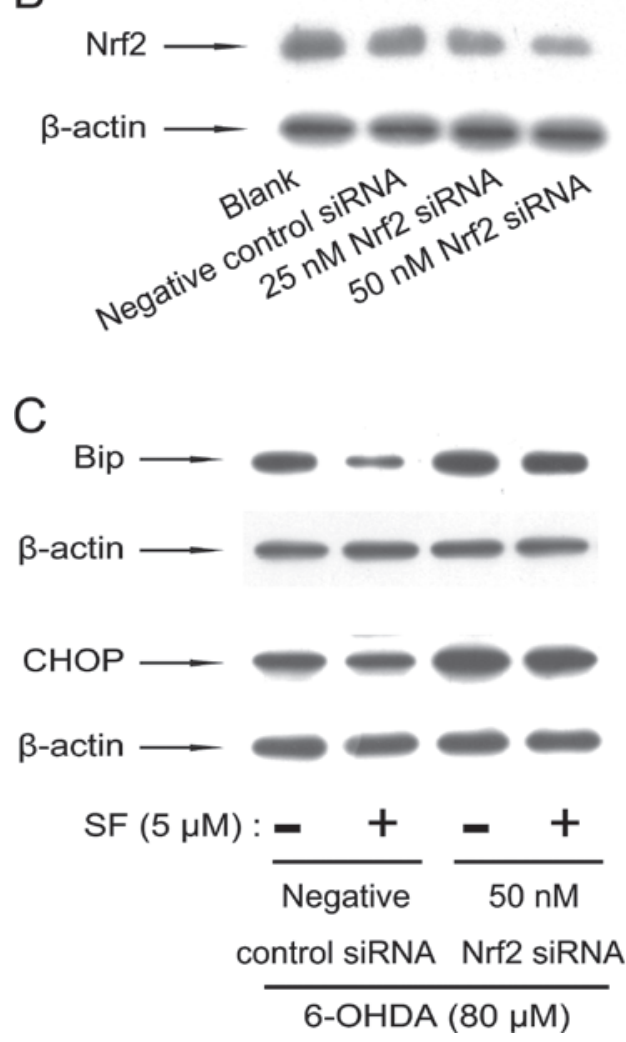

Figure 3. Effects of NF-E2-related factor-2 (Nrf2) on the protection of sulforaphane (SF) against 6-hydroxydopamine (6-OHDA)-induced endoplasmic reticulum (ER) stress in PC12 cells. (A) PC12 cells were treated with SF $(5 \mu \mathrm{M})$ for the indicated times and nuclear extracts were prepared for the western blot analysis of Nrf2. All experiments were performed in triplicate. (B) The Nrf2 protein levels were measured in PC12 cells (Blank) and PC12 cells were transfected with $N r f 2$ siRNA or negative control siRNA for $24 \mathrm{~h}$. (C) PC12 cells, transfected with Nrf2 siRNA or control siRNA for $24 \mathrm{~h}$, were treated with $5 \mu \mathrm{M} \mathrm{SF}$ for $6 \mathrm{~h}$ and then challenged with $80 \mu \mathrm{M}$ 6-OHDA. Cells were harvested and lysed, and an aliquot of protein was subjected to SDS-PAGE for western blot analysis, as described in Materials and methods. Representative bands are shown from triplicate experiments. CHOP, C/EBP homologous protein.

to its anti-oxidant properties, such as radical scavenging and the induction of anti-oxidant genes $(26,27)$. The Nrf2-ARE pathway is involved in the modulation of oxidative stress, inflammation and ER stress (28-31). It has been reported that the activation of the Nrf2-ARE pathway plays an important role in the various bioactivities of SF, suggesting that mechanisms other than anti-oxidant activities are involved in the underlying benefical effects of SF.

Previous studies have demonstrated that the activation of ER stress-mediated cell death is linked to PD $(2,3)$. To our knowledge, there have been no reports on the involvement of $\mathrm{SF}$ in the modulation of ER stress. ER stress response induced by 6-OHDA or other ER stress inducers is transmitted through 
the activation of three responsible proteins: inositol-required enzyme 1 (IRE1), protein kinase-like ER kinase (PERK) and activating transcription factor 6 (ATF6) (3). PERK phosphorylates and inactivates eIF $2 \alpha$ to reduce the protein load on the ER. These responses are followed by the increased expression of CHOP and subsequent apoptosis (32). In the present study, SF dramatically decreased the expression of early ER stress response proteins and subsequent $\mathrm{CHOP}$ expression by $6-O H D A$. These results suggest that SF protects against 6-OHDA-induced cell death partly by inhibiting ER stress.

The generation of reactive oxygen species (ROS) is an important mediator for 6-OHDA-induced neuronal death $(33,34)$. The production of ROS inevitably triggers stress-activated signaling pathways, including ER stress responses (35). It has been reported that anti-oxidants protect cells against ER stress (36). These results suggest that the antioxidant activity of SF may contribute to the inhibition of ER stress response. The mechanism underlying the anti-oxidant activity of SF relies on Nrf2 activation. Previous studies have shown that Nrf2 signaling plays a cytoprotective role in response to ER stress. The activation of Nrf2 is required for survival during UPR. Nrf2 ${ }^{-/-}$cells are sensitive to various ER stress-inducing agents, and the overexpression of Nrf2 enhances cell survival during UPR $(29,37)$. In the present study, we demonstrate that the inhibitory effect of SF on 6-OHDA-induced ER stress is associated with the activation of Nrf2. The transfection of Nrf2 siRNA reversed the inhibitory effects of SF on 6-OHDA-induced ER stress responses. The mechanism of Nrf2-mediated cytoprotection relies on the ability of Nrf2 target genes. Heme oxygenase-1 and thioredoxin, two important targets of Nrf2, play roles in modulating ER stress (38-40). These results suggest that SF prevents ER stress response by 6-OHDA through the activation of $\mathrm{Nrf} 2$. However, further studies are required to explore the target genes of Nrf2 involved in the inhibition of ER stress by SF.

In conclusion, our results indicate that SF has a cytoprotective effect against the neurotoxin, 6-OHDA, and that this protection may be mediated by the inhibitory effect of SF on 6-OHDA-induced ER stress. The regulation of ER stress by $\mathrm{SF}$ is associated with the activation of Nrf2. SF may be a novel neuroprotective agent against ER stress, as well as a therapeutic candidate for the treatment of ER stress-associated neural diseases, including Parkinson's disease.

\section{Acknowledgements}

This study was supported by the Science and Technology Joint Special Fund of Yunnan Province (2009CD199).

\section{References}

1. Pakkenberg B, Moller A, Gundersen HJ, Mouritzen Dam A and Pakkenberg H: The absolute number of nerve cells in substantia nigra in normal subjects and in patients with Parkinson's disease estimated with an unbiased stereological method. J Neurol Neurosurg Psychiatry 54: 30-33, 1991.

2. Ryu EJ, Harding HP, Angelastro JM, Vitolo OV, Ron D and Greene LA: Endoplasmic reticulum stress and the unfolded protein response in cellular models of Parkinson's disease. J Neurosci 22: 10690-10698, 2002.

3. Holtz WA and O'Malley KL: Parkinsonian mimetics induce aspects of unfolded protein response in death of dopaminergic neurons. J Biol Chem 278: 19367-19377, 2003.
4. Chen G, Bower KA, Ma C, Fang S, Thiele CJ and Luo J: Glycogen synthase kinase 3beta (GSK3beta) mediates 6-hydroxydopamineinduced neuronal death. FASEB J 18: 1162-1164, 2004.

5. Blum D, Torch S, Lambeng N, et al: Molecular pathways involved in the neurotoxicity of 6-OHDA, dopamine and MPTP: contribution to the apoptotic theory in Parkinson's disease. Prog Neurobiol 65: 135-172, 2001.

6. Hwang YP and Jeong HG: The coffee diterpene kahweol induces heme oxygenase-1 via the PI3K and p38/Nrf2 pathway to protect human dopaminergic neurons from 6-hydroxydopamine-derived oxidative stress. FEBS Lett 582: 2655-2662, 2008.

7. Kim I, Xu WJ and Reed JC: Cell death and endoplasmic reticulum stress: disease relevance and therapeutic opportunities. Nat Rev Drug Discov 7: 1013-1030, 2008.

8. Lindholm D, Wootz H and Korhonen L: ER stress and neurodegenerative diseases. Cell Death Differ 13: 385-392, 2006.

9. Rao RV, Ellerby HM and Bredesen DE: Coupling endoplasmic reticulum stress to the cell death program. Cell Death Differ 11: 372-380, 2004.

10. Harding HP, Zhang Y, Bertolotti A, Zeng H and Ron D: Perk is essential for translational regulation and cell survival during the unfolded protein response. Mol Cell 5: 897-904, 2000.

11. Boyce M, Bryant KF, Jousse C, et al: A selective inhibitor of eIF2alpha dephosphorylation protects cells from ER stress. Science 307: 935-939, 2005.

12. Li J, Ni M, Lee B, Barron E, Hinton DR and Lee AS: The unfolded protein response regulator GRP78/BiP is required for endoplasmic reticulum integrity and stress-induced autophagy in mammalian cells. Cell Death Differ 15: 1460-1471, 2008.

13. McCullough KD, Martindale JL, Klotz LO, Aw TY and Holbrook NJ: Gadd153 sensitizes cells to endoplasmic reticulum stress by down-regulating $\mathrm{Bc} 12$ and perturbing the cellular redox state. Mol Cell Biol 21: 1249-1259, 2001.

14. Zinszner H, Kuroda M, Wang X, et al: CHOP is implicated in programmed cell death in response to impaired function of the endoplasmic reticulum. Genes Dev 12: 982-995, 1998.

15. Oyadomari S, Koizumi A, Takeda K, et al: Targeted disruption of the Chop gene delays endoplasmic reticulum stress-mediated diabetes. J Clin Invest 109: 525-532, 2002.

16. Kim I, Xu W and Reed JC: Cell death and endoplasmic reticulum stress: disease relevance and therapeutic opportunities. Nat Rev Drug Discov 7: 1013-1030, 2008.

17. Mattson MP, Son TG and Camandola S: Viewpoint: mechanisms of action and therapeutic potential of neurohormetic phytochemicals. Dose Response 5: 174-186, 2007.

18. Tarozzi A, Morroni F, Merlicco A, et al: Sulforaphane as an inducer of glutathione prevents oxidative stress-induced cell death in a dopaminergic-like neuroblastoma cell line. J Neurochem 111: 1161-1171, 2009.

19. Ping Z, Liu W, Kang Z, et al: Sulforaphane protects brains against hypoxic-ischemic injury through induction of Nrf2-dependent phase 2 enzyme. Brain Res 1343: 178-185, 2010.

20. Zhang Y, Talalay P, Cho CG and Posner GH: A major inducer of anticarcinogenic protective enzymes from broccoli: isolation and elucidation of structure. Proc Natl Acad Sci USA 89: 2399-2403, 1992.

21. Jazwa A, Rojo AI, Innamorato NG, Hesse M, Fernandez-Ruiz J and Cuadrado A: Pharmacological targeting of the transcription factor nrf 2 at the basal ganglia provides disease modifying therapy for experimental parkinsonism. Antioxid Redox Signal 14: 2347-2360, 2011.

22. Han JM, Lee YJ, Lee SY, et al: Protective effect of sulforaphane against dopaminergic cell death. J Pharmacol Exp Ther 321: 249-256, 2007.

23. Deng C, Tao R, Yu SZ and Jin H: Sulforaphane protects against 6-hydroxydopamine-induced cytotoxicity by increasing expression of heme oxygenase-1 in a PI3K/Akt-dependent manner. Mol Med Report 5: 847-851, 2012.

24. Soane L, Li Dai W, Fiskum G and Bambrick LL: Sulforaphane protects immature hippocampal neurons against death caused by exposure to hemin or to oxygen and glucose deprivation. J Neurosci Res 88: 1355-1363, 2010.

25. Tanito M, Masutani H, Kim YC, Nishikawa M, Ohira A and Yodoi J: Sulforaphane induces thioredoxin through the antioxidant-responsive element and attenuates retinal light damage in mice. Invest Ophthalmol Vis Sci 46: 979-987, 2005.

26. Konwinski RR, Haddad R, Chun JA, et al: Oltipraz, 3H-1,2dithiole-3-thione, and sulforaphane induce overlapping and protective antioxidant responses in murine microglial cells. Toxicol Lett 153: 343-355, 2004. 
27. Wierinckx A, Breve J, Mercier D, Schultzberg M, Drukarch B and Van Dam AM: Detoxication enzyme inducers modify cytokine production in rat mixed glial cells. J Neuroimmunol 166: 132-143, 2005.

28. Cullinan SB, Zhang D, Hannink M, Arvisais E, Kaufman RJ and Diehl JA: Nrf2 is a direct PERK substrate and effector of PERKdependent cell survival. Mol Cell Biol 23: 7198-7209, 2003.

29. Cullinan SB and Diehl JA: PERK-dependent activation of Nrf2 contributes to redox homeostasis and cell survival following endoplasmic reticulum stress. J Biol Chem 279: 20108-20117, 2004.

30. Lee JM, Calkins MJ, Chan K, Kan YW and Johnson JA: Identification of the NF-E2-related factor-2-dependent genes conferring protection against oxidative stress in primary cortical astrocytes using oligonucleotide microarray analysis. J Biol Chem 278: 12029-12038, 2003.

31. Innamorato NG, Rojo AI, Garcia-Yague AJ, Yamamoto M, de Ceballos ML and Cuadrado A: The transcription factor Nrf2 is a therapeutic target against brain inflammation. J Immunol 181: 680-689, 2008.

32. Szegezdi E, Logue SE, Gorman AM and Samali A: Mediators of endoplasmic reticulum stress-induced apoptosis. EMBO Rep 7: 880-885, 2006.

33. Cohen G and Heikkila RE: The generation of hydrogen peroxide, superoxide radical, and hydroxyl radical by 6-hydroxydopamine, dialuric acid, and related cytotoxic agents. J Biol Chem 249: 2447-2452, 1974.
34. Linert W, Herlinger E, Jameson RF, Kienzl E, Jellinger K and Youdim MB: Dopamine, 6-hydroxydopamine, iron, and dioxygen - their mutual interactions and possible implication in the development of Parkinson's disease. Biochim Biophys Acta 1316: 160-168, 1996.

35. Cullinan SB and Diehl JA: Coordination of ER and oxidative stress signaling: the PERK/Nrf2 signaling pathway. Int J Biochem Cell Biol 38: 317-332, 2006.

36. Malhotra JD, Miao H, Zhang K, et al: Antioxidants reduce endoplasmic reticulum stress and improve protein secretion. Proc Natl Acad Sci USA 105: 18525-18530, 2008.

37. Cullinan SB, Gordan JD, Jin J, Harper JW and Diehl JA: The Keap1-BTB protein is an adaptor that bridges Nrf2 to a Cul3based E3 ligase: oxidative stress sensing by a Cul3-Keap1 ligase. Mol Cell Biol 24: 8477-8486, 2004.

38. Nakaso K, Yano H, Fukuhara Y, Takeshima T, Wada-Isoe K and Nakashima K: PI3K is a key molecule in the Nrf2-mediated regulation of antioxidative proteins by hemin in human neuroblastoma cells. FEBS Lett 546: 181-184, 2003.

39. Bai J, Nakamura H, Kwon YW, et al: Does thioredoxin-1 prevent mitochondria- and endoplasmic reticulum-mediated neurotoxicity of 1-methyl-4-phenyl-1,2,3,6-tetrahydropyridine? Antioxid Redox Signal 9: 603-608, 2007.

40. Kim HP, Pae HO, Back SH, et al: Heme oxygenase-1 comes back to endoplasmic reticulum. Biochem Biophys Res Commun 404 $1-5,2011$. 